\title{
Is higher dose always the right answer in stereotactic body radiation therapy for small hepatocellular carcinoma?
}

\author{
Kyung Hwa Lee, MD¹, Jeong II Yu, MD, PhD¹, Hee Chul Park, MD, PhD ${ }^{1,2}$, \\ Su Yeon Park, MS', Jung Suk Shin, MS'1, Eun Hyuk Shin, PhD'1, Sungkoo Cho, PhD', \\ Sang Hoon Jung, PhD'1, Young Yih Han, PhD', Do Hoon Lim, MD, PhD' \\ 'Department of Radiation Oncology, Samsung Medical Center, Sungkyunkwan University School of Medicine, Seoul; \\ ${ }^{2}$ Department of Medical Device Management and Research, Samsung Advanced Institute for Health Sciences and Technology, \\ Sungkyunkwan University, Seoul, Korea
}

Purpose: This study was conducted to compare clinical outcomes and treatment-related toxicities after stereotactic body radiation therapy (SBRT) with two different dose regimens for small hepatocellular carcinomas (HCC) $\leq 3 \mathrm{~cm}$ in size.

Materials and Methods: We retrospectively reviewed 44 patients with liver-confined HCC treated between 2009 and 2014 with SBRT. Total doses of $45 \mathrm{~Gy}(n=10)$ or $60 \mathrm{~Gy}(n=34)$ in 3 fractions were prescribed to the $95 \%$ isodose line covering $95 \%$ of the planning target volume. Rates of local control (LC), intrahepatic failure-free survival (IHFFS), distant metastasis-free survival (DMFS), and overall survival (OS) were calculated using the Kaplan-Meier method.

Results: Median follow-up was 29 months (range, 8 to 64 months). Rates at 1 and 3 years were $97.7 \%$ and $95.0 \%$ for LC, 97.7\% and $80.7 \%$ for 0 S, $76 \%$ and $40.5 \%$ for IHFFS, and $87.3 \%$ and $79.5 \%$ for DMFS. Five patients (11.4\%) experienced degradation of albumin-bilirubin grade, $2(4.5 \%)$ degradation of Child-Pugh score, and $4(9.1 \%)$ grade 3 or greater laboratory abnormalities within 3 months after SBRT. No significant difference was seen in any oncological outcomes or treatment-related toxicities between the two dose regimens.

Conclusions: SBRT was highly effective for local control without severe toxicities in patients with HCC smaller than $3 \mathrm{~cm}$. The regimen of a total dose of 45 Gy in 3 fractions was comparable to 60 Gy in efficacy and safety of SBRT for small HCC.

Keywords: Hepatocellular carcinoma, Stereotactic body radiotherapy, Dose fractionations

\section{Introduction}

Hepatocellular carcinoma (HCC) is the fifth most common cancer worldwide and the third most common cause of cancer mortality [1]. Curative treatment options for HCC include surgical resection, liver transplantation, and percutaneous ablation therapy such as radiofrequency ablation (RFA). However, these treatments are suitable in only a limited number of patients because of underlying liver function, limited accessibility to donor organs, or tumor size or location [2]. Transarterial chemoembolization (TACE) is widely used for patients who are not eligible for these curative treatment

Received 03 January 2018, Revised 10 February 2018, Accepted 19 March 2018.

Correspondence: Jeong II Yu, MD, and Hee Chul Park, MD, Department of Radiation Oncology, Samsung Medical Center, Sungkyunkwan University School of Medicine, 81 Irwon-ro, Gangnam-gu, Seoul 06351, Korea. Tel: +82-2-3410-2602, Fax: +82-2-34102619, E-mail: jeongil.yu@samsung.com, hee.ro.park@skku.edu

(c) This is an Open Access article distributed under the terms of the Creative Commons Attribution Non-Commercial License (http://creativecommons.org/ licenses/by-nc/4.0/) which permits unrestricted non-commercial use, distribution, and reproduction in any medium, provided the original work is properly cited.

www.e-roj.org 
options. Although some randomized trials and meta-analyses showed the effectiveness of TACE [3-6], local control rates of 50\%-70\% at 1 year are unsatisfactory compared with other curative options such as RFA or resection [7]. Stereotactic body radiotherapy (SBRT) is an emerging alternative option for patients with HCC that is inoperable or unsuitable for other locoregional therapies including RFA or TACE. Several retrospective and prospective studies of SBRT for HCC showed encouraging outcomes, with local control rates of 66\%-100\% and overall survival (OS) of 52\%-100\% at 2 or 3 years without severe toxicities [8-16]. Especially for small HCC, results of recent studies indicate very high local control rates of 96\%$100 \%$ and $0 S$ rates of $56 \%-76 \%$ [14-16].

Nonetheless, the total dose or fractionation schedule for SBRT of HCC varies considerably according to tumor size, underlying liver function, and institutional experience. In our institution, two main regimens of $45 \mathrm{~Gy}$ in 3 fractions and 60 Gy in 3 fractions were used for SBRT for HCC. The purpose of this study was to compare the clinical outcomes and treatment-related toxicities after SBRT with two different dose regimens for predominantly small-sized HCC of $3 \mathrm{~cm}$ or less.

\section{Materials and Methods}

\section{Patients}

This study was performed after approval by the Institutional Review Board of Samsung Medical Center (No. 2017-11156). We reviewed data for all patients treated with SBRT for localized HCC from August 2009 to October 2014. Eligibility criteria were: HCC not suitable for or refractory to surgery and other locoregional modalities such as RFA or TACE; HCC $3 \mathrm{~cm}$ or less across longest diameter and fewer than three synchronous lesions; adequate residual functional liver volume greater than $700 \mathrm{~mL}$; HCC confined to the liver without extrahepatic metastases; Eastern Cooperative Oncology Group (ECOG) score 0 to 1 ; age $\geq 20$ years; and Child-Pugh class A or B. Patients with double primary cancers $(n=4)$, who underwent transplant after SBRT $(n=1)$, or were lost to follow-up within 6 months after SBRT $(n=4)$ were excluded to ensure accurate analysis of outcomes. Patients with other previous treatments were accepted.

For all patients, diagnosis of HCC was based on (1) histological confirmation, or (2) characteristic tumor appearance in imaging studies with the presence of risk factors including chronic hepatitis B or C viral infection, cirrhosis, and increased alpha-fetoprotein (AFP) according to guidelines of the Korean Liver Cancer Study Group [17]. All cases were presented to a multidisciplinary liver tumor board including hepatologists, hepatic surgeons, radiation oncologists, and radiologists. All patients provided signed informed consent to be treated.

\section{Treatment}

All patients were educated and trained in controlling their respiration reproducibly and regularly using a visual prompting device before the beginning of a simulation, as previously described by Jung et al. [18] at the same institution. A personalized vacuum cushion facilitated patient immobilization in a supine position and four-dimensional (4D) computed tomography (CT) scanning was performed while the patient breathed with visual guidance from the simulation. The 4D CTs were sorted into 10 phase bins retrospectively.

Gross tumor volume (GTV) was defined as the arterial enhancing lesion with washout in the portal or delayed phase. Planning CT images were co-registered with pretreatment diagnostic or radiation therapy (RT)-planning magnetic resonance imaging (MRI) to better identify viable HCCs. Clinical target volume (CTV) was defined as a $5.0-6.0 \mathrm{~mm}$ margin for GTV, and internal target volume (ITV) was defined as the envelope of CTVs in the phase within a gating window of 40\%-60\%. Planning target volume (PTV) was determined from ITV by adding an isotropic setup margin of 7.0-8.0 mm. Organs at risk (OAR) included whole liver, normal liver (whole liver minus CTV), stomach, duodenum, small and large bowels, kidneys, spinal cord, heart and great vessels, skin, and chest walls. The maximum dose for a $700-\mathrm{mL}$ of normal liver was restricted to less than $21 \mathrm{~Gy}$ in 3 fractions. Other prescription doses and planning constraints for OAR were defined as in Supplementary Table S1.

A treatment plan using Pinnacle ${ }^{3}$ (Philips Healthcare, Fitchburg, WI, USA) was conducted with 5-6 photon fields with nominal energy $10 \mathrm{MV}$ using the end-exhale 4D CT image. A total dose of either 45 or $60 \mathrm{~Gy}$ was prescribed to the isodose line covering 95\% of the PTV. Mainly, 45 Gy was used when SBRT was started for HCC, escalating to $60 \mathrm{~Gy}$ when we did not observe severe toxicity from $45 \mathrm{~Gy}$ in several patients. All patients were treated with a Novalis Tx (Varian Medical Systems, Palo Alto, CA, USA) equipped with a real-time position management system with a gating technique using a duty cycle of 40\%-60\%. Image guidance was performed in two stages before administering each fraction. First, 3D bone matching was performed using cone-beam CT followed by 2D surrogate matching in the anterior-posterior and lateral directions at the end-exhale phase using an On-Board Imager 
(Varian Medical Systems). In most patients, iodized oil uptake around the target by previous TACE and diaphragm at the endexhale phase were used as a surrogate. Two or three fiducial markers were implanted under sonographic guidance and used for setup with some patients.

\section{Patient follow-up}

All patients were assessed 1 month after SBRT, every 3 months for 2 years, and every 4-6 months thereafter. Follow-up included clinical examination, contrast-enhanced MRI or CT scan, biochemical profiles, and tumor markers. Local failure was defined as recurrence within the PTV, intrahepatic failure was defined as recurrence at any site in the liver but outside of the PTV, and distant metastasis was defined as recurrent disease at any site outside the liver. Toxicity was evaluated according to the Common Terminology Criteria for Adverse Events (CTCAE) version 4.0. Radiation-induced hepatic toxicity was evaluated according to any decline in liver function using Child-Pugh score and albumin-bilirubin (ALBI) grade [19] within 3 months after SBRT. Scores were censored after other liver-directed therapies for further intrahepatic recurrences after SBRT.

\section{Statistical analysis}

Quantitative variables were described by median and range and qualitative variables by frequency and percentage. Comparisons between the group treated with total dose $45 \mathrm{~Gy}$ (45-Gy group) and the group treated with $60 \mathrm{~Gy}$ (60-Gy group) were by two-sample t-test, $\chi^{2}$ test, Fisher exact test, or MannWhitney test. Rates of local control, intrahepatic failure-free survival (IHFFS), distant metastasis-free survival (DMFS), and OS were calculated according to the Kaplan-Meier method. Log-rank test was used to determine statistical significance of survival differences. All statistical analyses were performed using SPSS version 23.0 (IBM Corporation, Armonk, NY, USA)) and $p$-values $<0.05$ were considered statistically significant.

\section{Results}

\section{Patient characteristics}

A total of 46 liver lesions that were irradiated in 44 patients were included in analyses. Patient and treatment characteristics are in Table 1. Median follow-up was 29 months (range, 8 to 64 months) with 44 patients with a single lesion and 2 patients with 2 separate synchronous lesions. Almost all patients had previously experienced multiple treatments for HCC up to 14 times. Among all evaluable patients, only one was previously untreated with newly diagnosed HCC. Of the remaining 43 patients, 23 had newly developed intrahepatic recurrences in previous untreated lesions and 20 had recurrences of previously treated lesions. The median number of previous treatments was four; however, despite a large number of previous treatments, moderate liver functions were retained, with Child-Pugh scores $A 5$ or $A 6$ for all patients.

The 45-Gy group had 10 patients and the 60-Gy group had 34. No difference in patient characteristics were observed between the two groups except for year of treatment; the proportion of patients treated before 2013 was significantly larger in the 45-Gy group than in the 60-Gy group (90\% vs. 3\%; $p<0.001)$.

\section{Survival and prognostic factors}

For 44 patients, 46 tumors were treated and individually evaluated. Local control rate was $97.7 \%$ at 1 year and $95.0 \%$ at 3 years (Fig. 1A) with two cases of local failure. All local failures occurred in the 60-Gy group and were associated with extrahepatic progression. The first case of local failure, a $2.4-\mathrm{cm}$ lesion in the right dome, was treated by $60 \mathrm{~Gy}$ in 3 fractions, achieving complete response at 6 months after SBRT. Recurrence occurred at the lower margin of PTV 10 months after treatment and metastatic lung nodules were detected at the same time (Fig. 2A and 2B). The second case, a $2.0-\mathrm{cm}$ lesion, also in the right dome, was similarly treated with $60 \mathrm{~Gy}$ in 3 fractions, achieving complete response after 3 months. Distant failure in the peritoneal cavity was detected at 6 months after treatment and infield failure around the upper margin of PTV was discovered 17 months after SBRT (Fig. 2C and 2D). The two patients were treated with sorafenib after diagnosis of distant failure. The patient in the first case died 24 months after SBRT, and the patient in the second case was alive with disease at last follow-up 24 months after SBRT.

The OS rate was $97.7 \%$ at 1 year and $80.7 \%$ at 3 years (Fig. 1B). Nine patients died during follow-up, and all deaths were related to cancer progression. Of these 9 patients, 4 had both intrahepatic and distant progression, 4 had only intrahepatic progression, and 1 had both infield and distant progression. At 1 and 3 years, respectively, IHFFS rates were 76\% and 40.5\% (Fig. 1C) and DMFS rates were 87.3\% and 79.5\% (Fig. 1D). Median progression-free survival was 13.0 months (95\% confidence interval, 7.4-18.6). Among the 26 patients with tumor progression, 21 had only outfield intrahepatic progression, and five had both outfield intrahepatic and distant failures (Supplementary Fig. S1).

No differences in survival outcomes between the 45- 
Table 1. Patients' characteristics

\begin{tabular}{|c|c|c|c|c|}
\hline & All $(n=44)$ & $45 \mathrm{~Gy}(\mathrm{n}=10)$ & $60 \mathrm{~Gy}(\mathrm{n}=34)$ & $p$-value \\
\hline Age (yr) & $65(47-78)$ & $59(47-73)$ & $65(49-78)$ & 0.076 \\
\hline Sex & & & & 0.289 \\
\hline Male & $35(80)$ & $9(90)$ & $26(76)$ & \\
\hline Female & $9(20)$ & $1(10)$ & $8(24)$ & \\
\hline Hepatitis etiology & & & & 0.250 \\
\hline B & $31(70)$ & $8(80)$ & $23(68)$ & \\
\hline C & $6(14)$ & $0(0)$ & $6(18)$ & \\
\hline Other & $7(16)$ & $2(20)$ & $5(15)$ & \\
\hline Treatment year & & & & $<0.001$ \\
\hline$<2013$ & $10(23)$ & $9(90)$ & $1(3)$ & \\
\hline$\geq 2013$ & $34(77)$ & $1(10)$ & $33(97)$ & \\
\hline TACE before SBRT & & & & 0.206 \\
\hline Yes & $34(77)$ & $9(90)$ & $25(74)$ & \\
\hline No & $10(23)$ & $1(10)$ & $9(26)$ & \\
\hline No. of previous treatments & & & & 0.422 \\
\hline$<7$ & $31(71)$ & $6(60)$ & $25(74)$ & \\
\hline$\geq 7$ & $13(29)$ & $4(40)$ & $9(26)$ & \\
\hline Tumor diameter (mm) & $14(8-28)$ & $14(8-27)$ & $14(8-28)$ & 0.382 \\
\hline$<10$ & $4(9)$ & $2(20)$ & $2(6)$ & \\
\hline$\geq 10$ and $<20$ & $30(68)$ & $6(60)$ & $24(71)$ & \\
\hline$\geq 20$ and $<30$ & $10(23)$ & $2(20)$ & $8(24)$ & \\
\hline No. of synchronous lesions & & & & 0.168 \\
\hline 1 & $42(95)$ & $8(80)$ & $34(100)$ & \\
\hline 2 & $2(5)$ & $2(20)$ & $0(0)$ & \\
\hline AFP (ng/mL) & $9.05(1.6-1,558.1)$ & $11.15(1.8-1,558.1)$ & $7.85(1.6-394.2)$ & 0.237 \\
\hline$<3.5$ & $12(27)$ & $2(20)$ & $10(29)$ & \\
\hline$\geq 3.5$ & $32(73)$ & $8(80)$ & $24(71)$ & \\
\hline Child-Pugh score & & & & 0.516 \\
\hline A5 & $38(86)$ & $8(80)$ & $30(88)$ & \\
\hline A6 & $6(14)$ & $2(20)$ & $4(12)$ & \\
\hline \multicolumn{5}{|l|}{ BCLC stage } \\
\hline 0 & $31(70)$ & $5(50)$ & $26(76)$ & \\
\hline$A$ & $11(25)$ & $3(30)$ & $8(24)$ & \\
\hline$B$ & $0(0.0)$ & $0(0)$ & $0(0)$ & \\
\hline C & $2(5)$ & $2(20)$ & $0(0)$ & \\
\hline Type of HCC & & & & 0.054 \\
\hline Newly developed in previous untreated lesion & $24(55)$ & $8(80)$ & $16(47)$ & \\
\hline Recurred in previous treated lesion & $20(45)$ & $2(20)$ & $18(53)$ & \\
\hline
\end{tabular}

Values are presented as median (range) or number (\%).

TACE, trans-arterial chemoembolization; SBRT, stereotactic body radiation therapy; AFP, alpha-fetoprotein; BCLC, Barcelona Clinic Liver Cancer; HCC, hepatocellular carcinoma.

Gy group and 60-Gy group were observed. The 2-year local control rate was higher in the 45-Gy group than the 60-Gy group but the difference was not significant (100\% vs. 93.7\%; $p=0.462$ ). The 60-Gy group tended to have higher 3-year IHFFS and OS, but without significance (Table 2). Tumor size less than $2 \mathrm{~cm}$ was a favorable factor for local control $(p=$ 0.041). Etiology of chronic hepatitis B, AFP level lower than
$3.5 \mathrm{IU} / \mathrm{mL}$, and recurrence in a previous treated lesion were favorable factors for intrahepatic control. AFP $<3.5 \mathrm{IU} / \mathrm{mL}$ was the only favorable factor for survival.

\section{Toxicity}

All scheduled treatments were completed without manifestations of acute toxicities during treatment. Five 
Table 2. Prognostic factors

\begin{tabular}{|c|c|c|c|c|c|c|c|}
\hline & $\begin{array}{c}\text { No. of } \\
\text { patients }\end{array}$ & $\begin{array}{c}3-y r \text { LC } \\
(\%)\end{array}$ & p-value & $\begin{array}{c}3-y r \text { IHFFS } \\
(\%)\end{array}$ & p-value & $\begin{array}{c}3-y r \text { OS } \\
(\%)\end{array}$ & $p$-value \\
\hline \multicolumn{8}{|l|}{ Age (yr) } \\
\hline$<60$ & 15 & 100.0 & \multirow[t]{2}{*}{0.297} & 26.7 & \multirow[t]{2}{*}{0.088} & 72.0 & \multirow[t]{2}{*}{0.065} \\
\hline$\geq 60$ & 29 & 92.4 & & 44.3 & & 87.2 & \\
\hline \multicolumn{8}{|l|}{ Sex } \\
\hline Male & 35 & 93.6 & \multirow[t]{2}{*}{0.444} & 31.8 & \multirow[t]{2}{*}{0.068} & 76.2 & \multirow[t]{2}{*}{0.121} \\
\hline Female & 9 & 100.0 & & 66.7 & & 100.0 & \\
\hline \multicolumn{8}{|l|}{ Hepatitis etiology } \\
\hline $\mathrm{B}$ & 31 & 93.1 & \multirow[t]{3}{*}{0.669} & 51.6 & \multirow[t]{3}{*}{0.047} & 85.5 & \multirow[t]{3}{*}{0.422} \\
\hline C & 6 & 100.0 & & 16.7 & & 80.0 & \\
\hline Others & 7 & 100.0 & & 14.3 & & 62.5 & \\
\hline \multicolumn{8}{|l|}{ No. of previous treatments } \\
\hline$<7$ & 31 & 96.3 & \multirow[t]{2}{*}{0.475} & 45.2 & \multirow[t]{2}{*}{0.065} & 87.0 & \multirow[t]{2}{*}{0.061} \\
\hline$\geq 7$ & 13 & 91.7 & & 23.1 & & 64.7 & \\
\hline \multicolumn{8}{|l|}{ Tumor diameter (mm) } \\
\hline$<10$ & 4 & 100.0 & \multirow[t]{3}{*}{0.041} & 25.0 & \multirow[t]{3}{*}{0.313} & 100.0 & \multirow[t]{3}{*}{0.780} \\
\hline$\geq 10$ and $<20$ & 30 & 100.0 & & 36.7 & & 77.7 & \\
\hline$\geq 20$ and $<30$ & 10 & 80.0 & & 46.7 & & 80.0 & \\
\hline \multicolumn{8}{|l|}{ RT dose (Gy) } \\
\hline 45 & 10 & 100.0 & \multirow[t]{2}{*}{0.462} & 30.0 & \multirow[t]{2}{*}{0.688} & 67.5 & \multirow[t]{2}{*}{0.513} \\
\hline 60 & 34 & 93.7 & & 39.2 & & 80.3 & \\
\hline \multicolumn{8}{|l|}{ AFP (ng/mL) } \\
\hline$<3.5$ & 12 & 100.0 & \multirow[t]{2}{*}{0.352} & 66.7 & \multirow[t]{2}{*}{0.035} & 100.0 & \multirow[t]{2}{*}{0.026} \\
\hline$\geq 3.5$ & 32 & 92.9 & & 27.5 & & 72.4 & \\
\hline \multicolumn{8}{|l|}{ Child-Pugh score } \\
\hline A5 & 38 & 94.4 & \multirow[t]{2}{*}{0.615} & 39.1 & 0.615 & 80.6 & 0.988 \\
\hline A6 & 6 & 100.0 & & 33.3 & & 83.3 & \\
\hline Type of HCC & & & & & & & \\
\hline Newly developed in previous untreated lesion & 24 & 91.1 & 0.202 & 20.8 & 0.003 & 71.4 & 0.065 \\
\hline Recurred in previous treated lesion & 20 & 100.0 & & 58.3 & & 94.7 & \\
\hline
\end{tabular}

LC, local control; IHFFS, intrahepatic failure-free survival; OS, overall survival; RT, radiation therapy; AFP, alpha-fetoprotein; HCC, hepatocellular carcinoma.

Table 3. Hepatic toxicity

\begin{tabular}{llcl}
\hline \multicolumn{1}{c}{ Toxicity parameter } & Grade 3 & Grade 4-5 & Normalized \\
\hline Laboratory tests & & & \\
Elevated transaminases & $0(0)$ & $0(0)$ & - \\
Hyperbilirubinemia & $0(0)$ & $0(0)$ & - \\
Hypoalbuminemia & $0(0)$ & $0(0)$ & $0 / 4$ \\
Decreased platelet count & $4(9.1)$ & $0(0)$ & - \\
Elevated alkaline phosphatase & $0(0)$ & - & $3 / 5$ \\
Degradation of ALBI grade & $5(11.4)$ & - & $1 / 2$ \\
Degradation of Child-Pugh score & $2(4.5)$ & & \\
\hline
\end{tabular}

Values are presented as number (\%).

$A L B I$, albumin-bilirubin.

patients (11.4\%) experienced a decline of one or more ALBI grade from baseline within 3 months of SBRT, while $2(4.5 \%)$ experienced worsening of Child-Pugh score by one or more within 3 months (Table 3). Four patients (9.1\%) experienced 
A

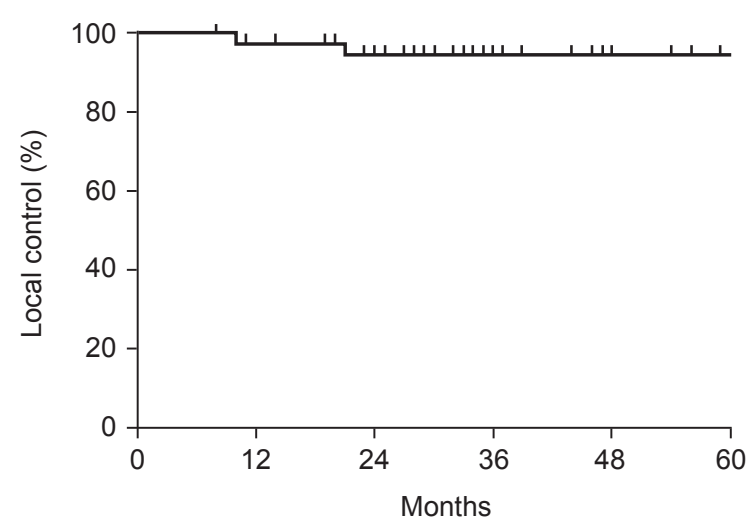

C

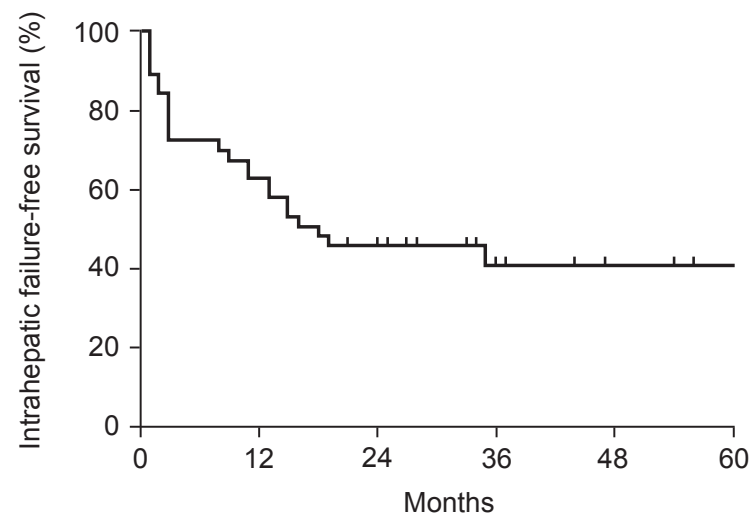

B

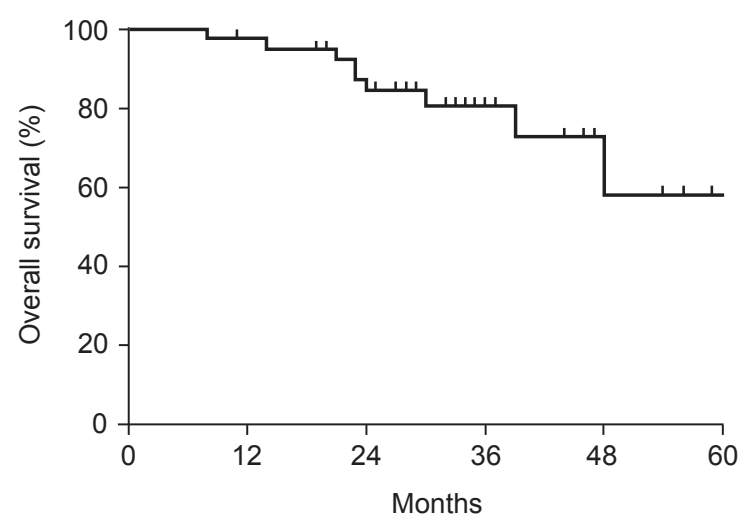

D

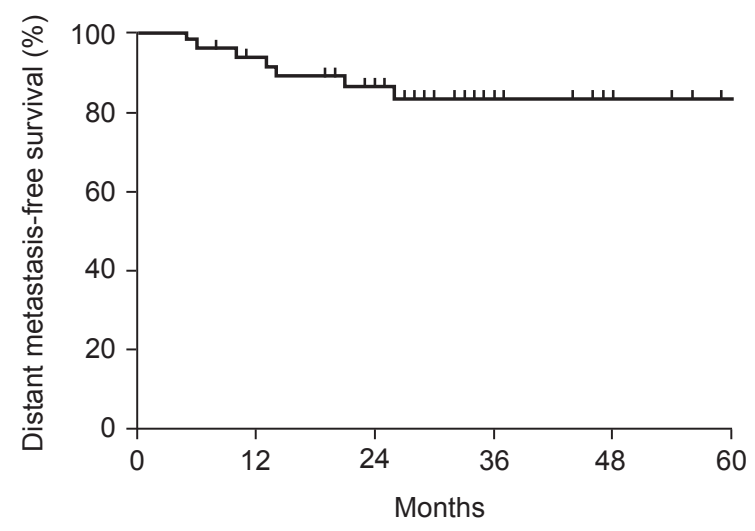

Fig. 1. Kaplan-Meier plots of (A) local control, (B) overall survival, (C) intrahepatic failure-free survival, and (D) distant metastasis-free survival.

a CTCAE grade 3 or greater laboratory score, specifically of decreased platelet count, with deterioration from baseline within 3 months after SBRT. In the 45-Gy group, degradation of ALBI grade was seen in one patient and grade 3 or greater laboratory score in one. In the 60-Gy group, degradation of ALBI grade was seen in 4 patients and of Child-Pugh score in two; grade 3 or greater laboratory score was seen in 3 patients. No significant difference in toxicity profiles was observed between two groups. Of 21 patients (48\%) who had esophagogastroduodenoscopy within 3 months after SBRT, only one had grade 1 gastric ulcers and two patients had grade 1 gastric erosions (Supplementary Table S2). These patients were all asymptomatic and no further treatments were needed. No other radiation-induced acute toxicities greater than grade 3 were reported.

\section{Discussion and Conclusion}

SBRT is an emerging treatment option for HCC that is inoperable or unsuitable for other curative locoregional modalities. Recent retrospective and prospective studies of small HCC lesions have shown promising outcomes for local control rate ranging from $96 \%-100 \%$, and OS rates ranging from $56 \%$ to $76 \%$ with acceptable toxicities [14-16]. However, studies had considerable heterogeneity among institutions for indications, total dose and fractionation schedules, and methods of respiratory motion control.

For small residual or recurrent HCC that is unsuitable for treatment by other locoregional modalities, our institution uses a salvage hypofractionated radiotherapy regimen of 50 or 60 Gy over 10 fractions before SBRT [20,21]. In this setting, at 3 years, the local control rate was $89.7 \%$ and the OS rate $57.4 \%$ with no grade 3 or greater treatment-related toxicities Directly comparing these results with our current SBRT results is difficult because the patient groups were different. However, considering that the indication and aim of hypofractionated radiotherapy were similar, our current study showed an improved local control rate with similar toxicity using our current SBRT regimen.

We mainly used a dose regimen of 45 Gy per 3 fractions, escalating to 60 Gy per 3 fractions after we observed no severe toxicity in several cases using $45 \mathrm{~Gy}$. The background 


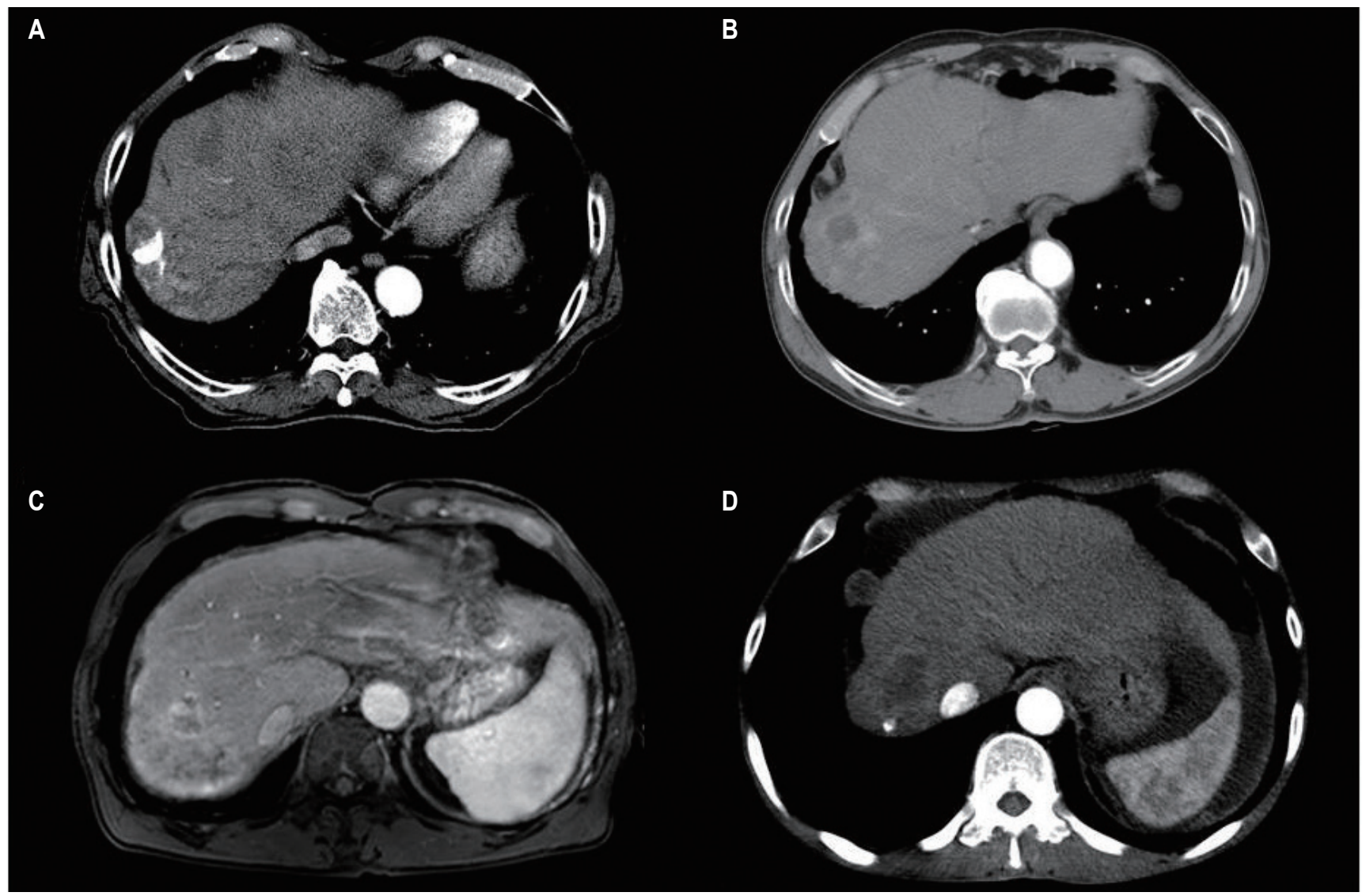

Fig. 2. Locally failed tumor characteristics. (A) Pretreatment computerized tomography (CT) image of a 2.4-cm lesion in the right dome area. (B) CT image when local failure was detected 10 months after treatment. (C) Pretreatment magnetic resonance imaging of a $2.0-\mathrm{cm}$ lesion in the right dome area. (D) CT image when local failure was detected 17 months after treatment.

of dose escalation was to improve the local control rate under acceptable toxicity; however, no difference in any clinical outcomes were seen between the 45-Gy group and 60-Gy group. Even, the two cases of local failure were observed in the 60-Gy group. One possible cause of local failures despite higher doses might be a radioresistant tumor biology related to low vascularity or dedifferentiated tumor nature. In both patients, tumors appeared with low signal intensities with peripheral enhanced rims in the arterial phase of planning $\mathrm{MRI}$, and they appeared as increased low-attenuation lesions at diagnosis of local failure (Fig. 2), supporting our hypothesis. Additionally, distant failures were found in both patients around or before diagnosis of local failure, which was distinct from other patients in whom the most common failure pattern was intrahepatic failure. This finding might be indicative of aggressive features in the two patients. We also found that radiation targeting lesions in both patients were sufficiently covered by focal liver reactions to appear in follow-up MRI at 3-4 months after SBRT. This finding suggested that physical or marginal issues contributed less to local failures.

Different institutions use widely different total doses and fractionation regimens for treating HCC with SBRT. Dose regimens and corresponding biologically equivalent doses (BEDs) using $\alpha / \beta$ ratio of 10 with local control rates for previous studies and our study are in Table 4. Explaining these various results using a simple rule is difficult. However, in general, a small or single HCC smaller than $3 \mathrm{~cm}$ was likely to be controlled well using a BED greater than 100 Gy. Yoon et al. [12] demonstrated that most local failures are found in patients with $\mathrm{HCCS}>3 \mathrm{~cm}$ with a local control rate at 3 years of $76.3 \%$ in patients with HCCs $>3 \mathrm{~cm}, 93.3 \%$ in patients with tumors between 2.1 and $3 \mathrm{~cm}$, and 100\% in patients with tumors $\leq 2 \mathrm{~cm}$ in their study using a median total dose of $45 \mathrm{~Gy}$ in 3 fractions. Takeda showed the possibility of an acceptable local control rate of $96.3 \%$ at 3 years for small ( $\leq 4$ $\mathrm{cm}$ ) and single HCCs using BEDs less than 72 Gy with optional TACE [16]. Studies by Bujold et al. [11] and Scorsetti et al. [13] using BEDs lower than 100 Gy showed lower local control 
Table 4. Other studies

\begin{tabular}{|c|c|c|c|c|c|c|}
\hline Author, year & $\begin{array}{c}\text { No. of } \\
\text { patients }\end{array}$ & $\begin{array}{l}\text { Tumor size } \\
(\mathrm{mm})\end{array}$ & Dose (Gy)/fx & BED (Gy) & $2-y r$ LC (\%) & $2-y r$ OS (\%) \\
\hline Kwon et al. [8], 2010 & 42 & $2.5(1.4-4.3)$ & $33 / 3$ & 69.3 & $3-y r: 67.5$ & $3-y r: 58.6$ \\
\hline Andolino et al. [9], 2011 & 60 & $3.1(1.0-6.5)$ & $\begin{array}{l}44 / 3(60) \\
40 / 5(40)\end{array}$ & $\begin{array}{c}108.9(60) \\
72(40)\end{array}$ & 90 & 67 \\
\hline Kang et al. [10], 2012 & 47 & $2.9(1.3-7.8)$ & $57 / 3$ & 165.3 & 94.6 & 68.7 \\
\hline Bujold et al. [11], 2013 & 102 & $7.2(1.4-23.1)$ & $36 / 6$ & 57.6 & $1-y r: 87$ & Median: 17 mo \\
\hline Yoon et al. [12], 2013 & 93 & $2.0(1.0-6.0)$ & $45 / 3$ & 112.5 & $3-y r: 92.1$ & $3-y r: 53.8$ \\
\hline Scorsetti et al. [13], 2015 & 43 & $4.8(1-12.5)$ & $\begin{array}{l}48-75 / 3(48) \\
36-60 / 6(52)\end{array}$ & $\begin{array}{c}124.8-262.5(48) \\
57.6-120(52)\end{array}$ & 64.4 & 45.3 \\
\hline Huertas et al. [14], 2015 & 77 & $2.4(0.7-6.3)$ & $45 / 3$ & 112.5 & 99 & 56.6 \\
\hline Kimura et al. [15], 2015 & 65 & $1.6(0.5-5.4)$ & $48 / 4$ & 105.6 & 100 & 76.0 \\
\hline Takeda et al. [16], 2016 & 90 & $2.3(1.0-4.0)$ & $\begin{array}{l}35 / 5(11) \\
40 / 5(89)\end{array}$ & $\begin{array}{l}59.5(11) \\
72.0(89)\end{array}$ & $3-y r: 96.3$ & $3-y r: 66.7$ \\
\hline Current study & 44 & $1.4(0.8-5.2)$ & $\begin{array}{l}45 / 3(23) \\
60 / 3(77)\end{array}$ & $\begin{array}{c}112.5(23) \\
180(77)\end{array}$ & $3-y r: 95$ & $3-y r: 80.7$ \\
\hline
\end{tabular}

Values are presented as median (range) or number (\%).

BED, biologically equivalent dose; LC, local control; OS, overall survival; RTOG, Radiation Therapy Oncology Group; CTCAE, Common Terminology Criteria for Adverse Events; CPS, Child-Pugh score; ALBI, albumin-bilirubin.

rates, although most patients in their studies had relatively large or multiple HCCs. The association between local control rates and BEDs of previous studies is in Supplementary Fig. S2. The trend was for local control rates to be more than $90 \%$ using BEDs above $100 \mathrm{~Gy}$, but no linear associations were seen above $100 \mathrm{~Gy}$. Considering these results and our experiences, higher total doses do not seem to guarantee higher local control rates. However, further studies are warranted to find proper doses and fraction schedules for SBRT for HCCS considering tumor size, location and biological features.

There are a variety of methods to investigate toxicity after SBRT such as classic radiation-induced liver disease (RILD), non-classic RILD, CTCAE grade, degradation of Child-Pugh score, and the recently introduced ALBI grade. Most studies use incidence of CTCAE grade 3 or more and worsening of ChildPugh score within 3-6 months after SBRT to describe hepatic toxicities. In the literature, incidence of CTCAE grade 3 or more is reported to be $2.4 \%-30 \%$ and incidence of worsening ChildPugh score is 4.7\%-46\%. Explanations for these diverse results for hepatic toxicities after SBRT include differences in tumor size, dose regimen, retained liver function at treatment, and irradiated volume of normal liver. Some studies show a strong correlation between some SBRT dosimetric parameters and hepatic toxicities [22-24]. Our investigated any degradation of ALBI grade and Child-Pugh score, and grade 3 or greater adverse events within 3 months after SBRT to evaluate toxicity. We examined if differences were detectable between the two dose regimens. Five patients (11.4\%) experienced degradation of ALBI grade, two had (4.5\%) degradation of Child-Pugh score, and four (9.1\%) had grade 3 or greater adverse events. No significant difference was observed in these parameters between the 45-Gy group and 60-Gy group. This low incidence of hepatic toxicities compared with other studies might be explained by the small tumor sizes and previous good liver functions within Child-Pugh score $A$ in all patients. Additionally, the small number of patients, low incidence of hepatic toxicities, and individual dosimetric parameters might have contributed to the small difference between the two dose groups despite the large difference in BEDs.

In our institution, the majority of patients were heavily treated with modalities such as TACE, RFA, local resection, and radiation therapy; the maximum number of treatments was 14 in one patient who was treated 9 times with TACE and 5 times with RFA before SBRT. However, all patients in this study had moderate liver function with Child Pugh score A5 to A6 despite multiple previous treatments. Our results indicated if patients have relevant liver function, especially Child-Pugh score $A$, the outcomes and toxicity of SBRT for small HCCs could be acceptable, even with a history of multiple treatments.

The limitations of this study were mainly the retrospective design and relatively small number of patients. However, this study was planned to compare two dose groups with no other differences in patient characteristics except treatment time; all were treated with the same treatment techniques in a single 
institution. Local control rates after SBRT for small HCCs in this study were highly compatible with results of other studies [14-16].

In conclusion, SBRT was highly effective for local control without severe toxicity in patients with HCCs smaller than 3 $\mathrm{cm}$. This treatment should be considered as an alternative and effective treatment option for small HCCs that are unsuitable for resection or other locoregional modalities. No significant difference was observed in oncological outcomes, including local control rate or overall survival, or hepatic toxicities after SBRT between groups treated by a total dose of $45 \mathrm{~Gy}$ in 3 fractions versus $60 \mathrm{~Gy}$ in 3 fractions. The regimen of a total dose of $45 \mathrm{~Gy}$ in 3 fractions was comparable to $60 \mathrm{~Gy}$, which appeared to be a reasonable option for SBRT in small HCC.

\section{Conflict of Interest}

No potential conflict of interest relevant to this article was reported.

\section{Supplementary Materials}

Supplementary materials can be found via https://doi. org/10.3857/roj.2017.00598. Table S1. Constraints to organs at risk. Table S2. Gastrointestinal and other toxicities (CTCAE grade). Fig. S1. Failure pattern related to radiation therapy target volume. Fig. S2. Local control (LC) rates related to biologically equivalent dose (BED) in other studies.

\section{References}

1. El-Serag HB, Rudolph KL. Hepatocellular carcinoma: epidemiology and molecular carcinogenesis. Gastroenterology 2007;132:2557-76.

2. Bruix J, Sherman M; Practice Guidelines Committee, American Association for the Study of Liver Diseases. Management of hepatocellular carcinoma. Hepatology 2005;42:1208-36.

3. Camma C, Schepis F, Orlando A, et al. Transarterial chemoembolization for unresectable hepatocellular carcinoma: meta-analysis of randomized controlled trials. Radiology 2002;224:47-54.

4. Lo CM, Ngan H, Tso WK, et al. Randomized controlled trial of transarterial lipiodol chemoembolization for unresectable hepatocellular carcinoma. Hepatology 2002;35:1164-71.

5. Llovet JM, Real MI, Montana $X_{1}$ et al. Arterial embolisation or chemoembolisation versus symptomatic treatment in patients with unresectable hepatocellular carcinoma: a randomised controlled trial. Lancet 2002;359:1734-9.

6. Llovet JM, Bruix J. Systematic review of randomized trials for unresectable hepatocellular carcinoma: chemoembolization improves survival. Hepatology 2003;37:429-42.

7. Oliveri RS, Wetterslev J, Gluud C. Transarterial (chemo) embolisation for unresectable hepatocellular carcinoma. Cochrane Database Syst Rev 2011 ;(3):CD004787.

8. Kwon JH, Bae SH, Kim JY, et al. Long-term effect of stereotactic body radiation therapy for primary hepatocellular carcinoma ineligible for local ablation therapy or surgical resection: stereotactic radiotherapy for liver cancer. BMC Cancer 2010;10:475.

9. Andolino DL, Johnson CS, Maluccio M, et al. Stereotactic body radiotherapy for primary hepatocellular carcinoma. Int J Radiat Oncol Biol Phys 2011;81:e447-53.

10. Kang JK, Kim MS, Cho CK, et al. Stereotactic body radiation therapy for inoperable hepatocellular carcinoma as a local salvage treatment after incomplete transarterial chemoembolization. Cancer 2012;118:5424-31.

11. Bujold A, Massey CA, Kim JJ, et al. Sequential phase I and II trials of stereotactic body radiotherapy for locally advanced hepatocellular carcinoma. J Clin Oncol 2013;31:1631-9.

12. Yoon SM, Lim YS, Park MJ, et al. Stereotactic body radiation therapy as an alternative treatment for small hepatocellular carcinoma. PLoS One 2013;8:e79854.

13. Scorsetti $M$, Comito $T$, Cozzi $L$, et al. The challenge of inoperable hepatocellular carcinoma (HCC): results of a singleinstitutional experience on stereotactic body radiation therapy (SBRT). J Cancer Res Clin Oncol 2015;141:1301-9.

14. Huertas A, Baumann AS, Saunier-Kubs F, et al. Stereotactic body radiation therapy as an ablative treatment for inoperable hepatocellular carcinoma. Radiother Oncol 2015;115:211-6.

15. Kimura T, Aikata H, Takahashi S, et al. Stereotactic body radiotherapy for patients with small hepatocellular carcinoma ineligible for resection or ablation therapies. Hepatol Res 2015:45:378-86.

16. Takeda A, Sanuki N, Tsurugai Y, et al. Phase 2 study of stereotactic body radiotherapy and optional transarterial chemoembolization for solitary hepatocellular carcinoma not amenable to resection and radiofrequency ablation. Cancer 2016;122:2041-9.

17. Korean Liver Cancer Study Group (KLCSG); National Cancer Center, Korea (NCC). 2014 Korean Liver Cancer Study GroupNational Cancer Center Korea practice guideline for the management of hepatocellular carcinoma. Korean J Radiol 2015;16:465-522.

18. Jung SH, Yu JI, Park HC, Lim DH, Han Y. A feasibility study 
evaluating the relationship between dose and focal liver reaction in stereotactic ablative radiotherapy for liver cancer based on intensity change of Gd-EOB-DTPA-enhanced magnetic resonance images. Radiat Oncol J 2016;34:64-75.

19. Johnson PJ, Berhane S, Kagebayashi $C$, et al. Assessment of liver function in patients with hepatocellular carcinoma: a new evidence-based approach-the ALBI grade. J Clin Oncol 2015;33:550-8.

20. Bae SH, Park HC, Lim DH, et al. Salvage treatment with hypofractionated radiotherapy in patients with recurrent small hepatocellular carcinoma. Int J Radiat Oncol Biol Phys 2012;82:e603-7.

21. Yu JI, Park HC, Lim DH, Paik SW. Do biliary complications after hypofractionated radiation therapy in hepatocellular carcinoma matter? Cancer Res Treat 2016;48:574-82.

22. Lasley FD, Mannina EM, Johnson CS, et al. Treatment variables related to liver toxicity in patients with hepatocellular carcinoma, Child-Pugh class A and B enrolled in a phase 1-2 trial of stereotactic body radiation therapy. Pract Radiat Oncol 2015;5:e443-e449.

23. Toesca DA, Osmundson EC, von Eyben $R$, Shaffer JL, Koong $A C$, Chang DT. Assessment of hepatic function decline after stereotactic body radiation therapy for primary liver cancer. Pract Radiat Oncol 2017;7:173-82.

24. Velec M, Haddad CR, Craig T, et al. Predictors of liver toxicity following stereotactic body radiation therapy for hepatocellular carcinoma. Int J Radiat Oncol Biol Phys 2017;97:939-46. 